Lepr Rev (1996) 67, 1-3

\title{
Editorial
}

\section{IS THERE A DECLINE IN LEPROSY PUBLICATIONS AND RESEARCH?}

Concern has been expressed recently that it is becoming more difficult to secure funds for leprosy research, particularly basic biomedical research as opposed to clinical or health services research. It is hard to say whether this is true or not as funding agencies will often turn the issue around and affirm that there are funds for good research suggesting that it is the researchers themselves who are turning to new areas. The background to this discussion is the success of the global MDT programme ${ }^{1}$ and the question of the need for further basic research. There is evidence that chemotherapy research is still very active with new drugs and new regimens under investigation. ${ }^{2}$

One approach to addressing whether or not there has been a reduction in research is to review the leprosy publications in the literature. Electronic databases such as Medline make this task possible. ${ }^{3}$ Medline is an electronic system covering the international literature on biomedicine, including the allied health fields and the biological and physical sciences, humanities, and information science as they relate to medicine and health care. Medline indexes information from approximately 3600 journals published worldwide including Leprosy Review, International Journal of Leprosy, Indian Journal of leprosy, Acta Leprologica and the Japanese Journal of Leprosy. It is produced by the National Library of Medicine and is updated monthly.

A count of the number of publications indexed based on the keyword 'leprosy' from 1980 to 1994 is shown in Figure 1. The pattern shown reveals a decline in the total number of leprosy publications (all languages) from 1991 to 1994, this was preceded by a rise from 1984 to 1988 . There is no evidence of peaks of publications associated with the International Leprosy Congresses $(1984,1988,1993)$ which might have been expected. The leprosy publications in English follow the same pattern as that of all languages, however the pattern of publications in the Abridged Index Medicus (AIM), which is a restricted set of 119 core clinical journals, shows a more sporadic pattern. The pattern of publications in tuberculosis, using the same method and over the same time period, shows an increasing trend from about 1984 (Figure 2).

This count of publications does tend to indicate a reduction in leprosy publications back to the levels of the mid-1980s, however, the current trend may yet continue downwards. Research publication numbers is not the same as research 


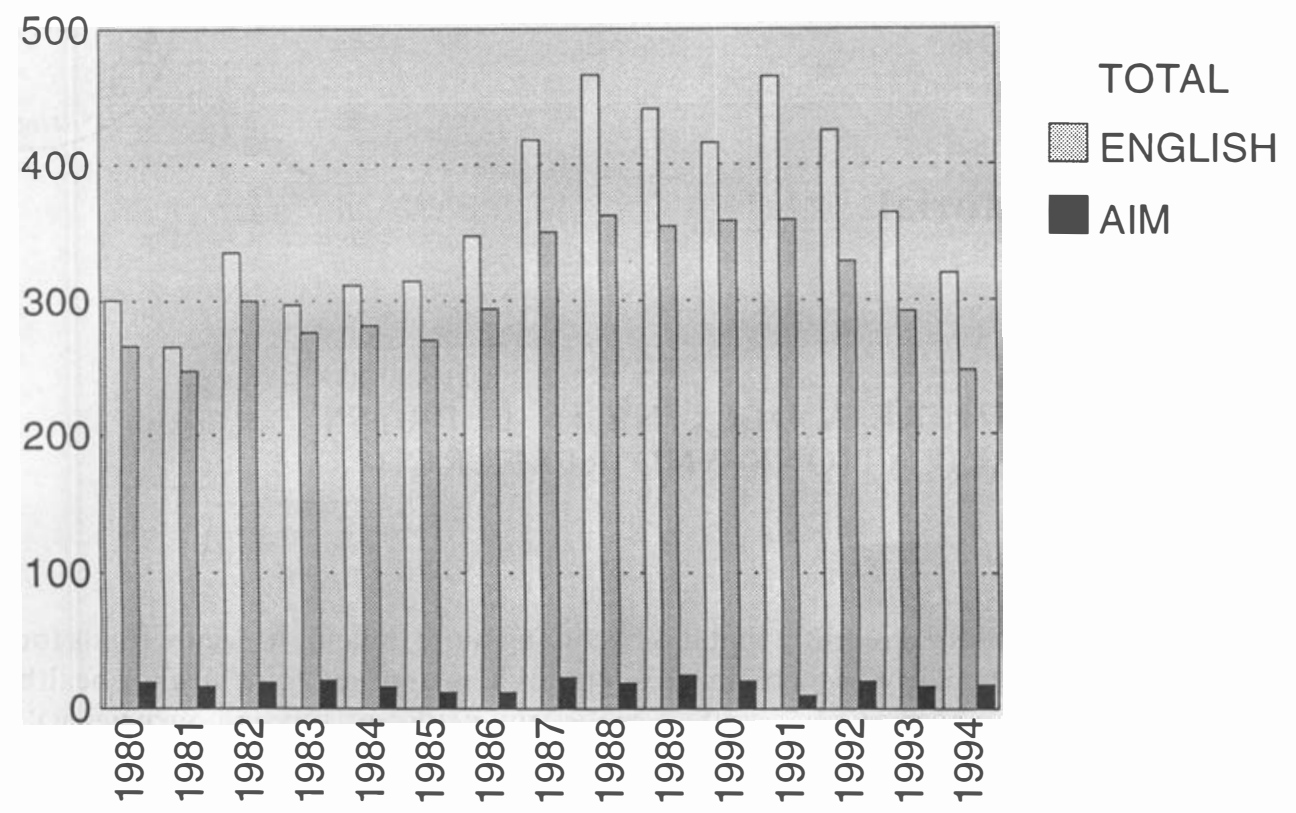

Figure 1. Leprosy publications, Medline 1980-94.

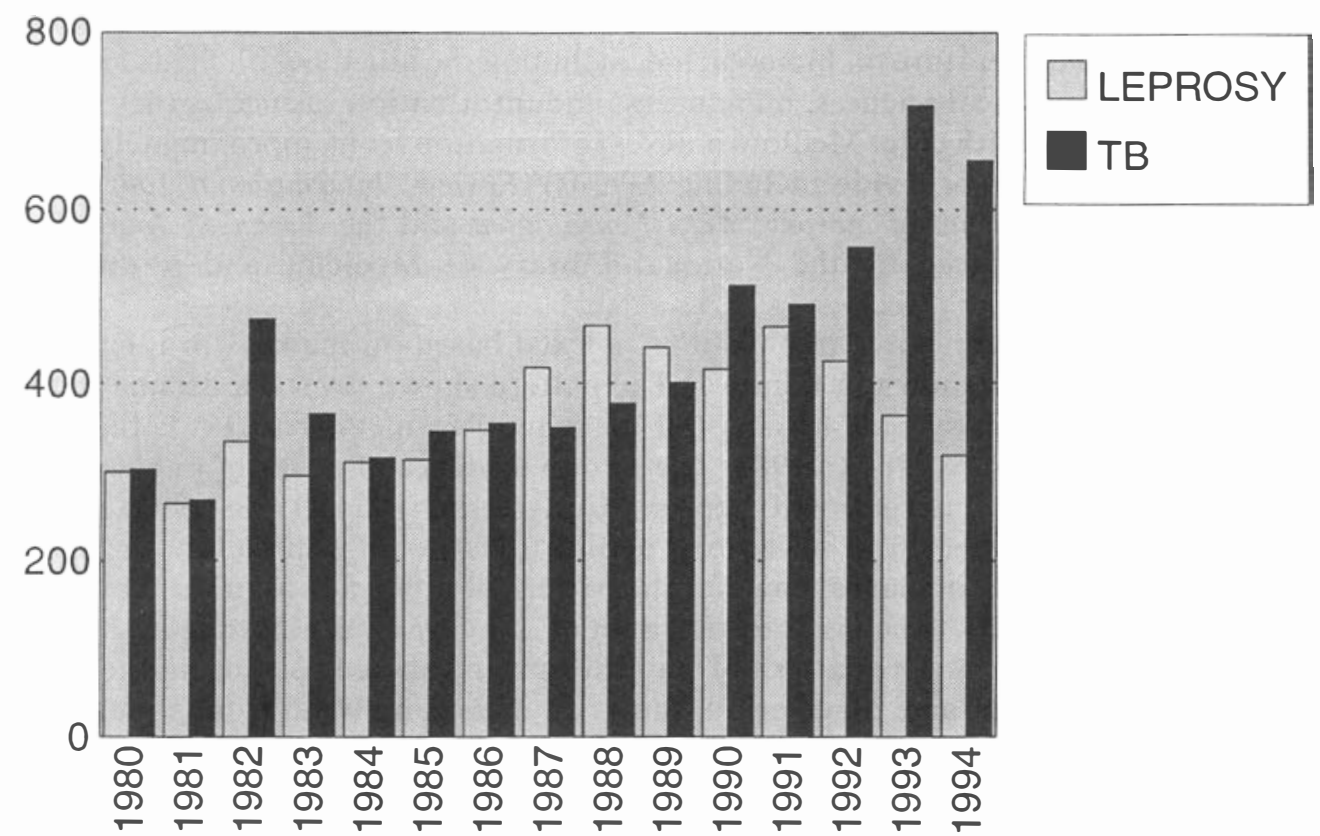

Figure 2. Leprosy and tuberculosis publications, Medline 1980-94. 
funding and would follow sometime after research funding. The decline in numbers of publications may also reflect the trend towards quality rather than quantity in publications. More important than concern about levels of funding and research is the issue of identifying what are the key research questions in leprosy which need to be addressed. As the global leprosy situation changes new questions need to be addressed and new technologies applied to old questions. The many unresolved questions about transmission, early infection and the development of protective immunity become increasingly important in post-elimination considerations of moving to eradication. The pathogenesis and natural history of nerve damage as well as its early detection and effective treatment are still areas of need for research.

Clinical research and health services research are less demanding of financial resources and reduced workload in many programmes as a result of the implementation of MDT should give more time for programme staff to engage in research or to write up for publication work recently undertaken. Research is needed into the relationship between case detection and true incidence rates, into disability assessment and prevention, into the needs for and the most effective means of rehabilitation. I am sure editors would want to see more publications rather than less. Major progress in leprosy has been achieved in the last 10 years, research needs are changing but the work which still needs to be done should not be underestimated. Research should be paving the way to providing more effective treatment and rehabilitation for leprosy patients and for tackling the long-term sequelae of leprosy infection, as well as the possibility of leprosy eradication.

University of Aberdeen

W. CAIRNS SMITH

Department of Public Health

Medical School Polwarth Building

Foresterhill, Aberdeen AB9 2ZD

Scotland, UK

\section{References}

1 World Health Organization. Progress towards the elimination of leprosy a s a public health problem. Wkly Epidemiol Rec 1995; 25: 177-82.

2 Grosset JH. Progress in the chemotherapy of leprosy. Int. J Lepr 1994; 62: 268-77.

3 Dickersin K, Scherer R, Lef ebvre C. Identif ying relevant studies for systematic reviews. Br Med J 1994; 309: $1286-91$ 